adjusting for smoking and lung cancer risk factors identified using directed acyclic graphs. The ORs were then pooled using a random-effects model.

Results Compared with low occupational PA as reference, subjects with medium and high occupational PA had ORs of 0.64 (95\% CI: $0.38-1.07)$ and 0.73 (95\% CI: 0.39-1.34), respectively. The ORs were similar by sex and by smoking status (never vs. ever smokers).

Conclusion Our findings suggest that, like recreational PA, occupational PA may protect against lung cancer risk.

\section{0-415 CHANGING TRENDS FOR MESOTHELIOMA IN CANADA AND THEIR IMPLICATIONS}

${ }^{1}$ Paul Demers, Hunter Warden, Lillian Tamburic, Catherine Slavik, Chris McLeod. 'Ontario Health, Canada

\subsection{6/OEM-2021-EPI.79}

Introduction Canada was once the world's largest producer of asbestos, but exposure has been decreasing since the 1970's due to restrictions on use, lower occupational exposure limits, closing of mines, and a ban in 2018 .

Objectives The objectives of this study were to evaluate how rates of mesothelioma in Ontario and British Columbia (BC), which together constitute over 50\% of the Canadian population, have changed over time, by sex, age, geographical region and tumour site.

Methods The Ontario and BC Cancer Registries were used to identify 4,146 and 1,659 malignant mesothelioma cases between the years 1993-2017 and 1992-2016, respectively. Time trends were examined by sex, age, and anatomical site. Birth cohort models for Ontario were fit using US National Cancer Institute's age-period-cohort analysis web tool.

Results Ontario incidence rates for mesothelioma climbed from $1.0 / 100,000$ in 1993 until 2012 when rates plateaued at approximately 1.6. In BC the rate climbed from 1.1 in 1993 to $1.7 / 100,000$ in 2003 , when it began to plateau. Although female rates are much lower than male, they continue to steadily rise in both provinces. Rates among people over the age of 70 rose dramatically over time, while rates were steady or dropped among people below the age of 50 in both provinces. Peritoneal rates continue to rise in Ontario, but not BC. Relative to the 1921-25 birth cohort, male incidence rate ratios increased until peaking in 1936-40. Rate ratios for subsequent male cohorts decreased. In contrast, using the same reference period, the risk in women rose slowly with successive birth cohorts, though confidence limits were wide due to the low case counts.

Conclusion These complex changes over time may be due to major reductions in exposure in the 1970's, longer latency periods associated with lower levels of exposure, and the growing importance of environmental exposures.

\section{Methods}

\section{0-15 OCCUPATIONAL HEALTH: A MULTI-COHORT JOB TITLE CLEANING PROJECT BY ALGORITHM}

'Ellen Sweeney, Christopher Baker, Mohammad Sadnan Al Manir, Deobrah Addey, Yunsong Cui, Hicks Jason, Cheryl Peters, Grace Shen Tu, Jennifer Vena, Anil Adisesh. ${ }^{1}$ Dalhousie University, Canada

10.1136/OEM-2021-EPI.80
Introduction Occupational data in prospective cohort studies is often underutilized due to the human and financial resources required to code open-ended text, such as job titles. Recognizing the value of occupational data in health research, as well as potential errors associated with manual coding, an Automated Coding Algorithm (ACA)-NOC algorithm was developed utilizing a Natural Language Processing approach.

Objectives We tested the ACA-NOC algorithm on two regional cohorts of a pan-Canadian cohort study, which represents the largest dataset an algorithm of this kind has been applied to. This process will harmonize and greatly expand the utility of the occupational data, enrich the research platforms, and further refine the efficiency of the algorithm.

Methods The ACA-NOC algorithm was tested on data from the Canadian Partnership for Tomorrow's Health (CanPath), a longitudinal cohort examining the role of genetic, environmental, lifestyle, and behavioural factors in the development of cancer and chronic disease. Using an iterative and interactive approach, the algorithm was applied to job title data from 111,000 questionnaires from two regional cohorts, coding the data to the Canadian National Occupation Classification (NOC) system. The algorithm was further refined based on each round of analysis, increasing the quantity of accurately coded data.

Results Results from this research demonstrate the ability to refine the ACA-NOC algorithm with a $10 \%$ overall improvement in exact matching from the baseline algorithm. There were also instances where the algorithm performance was superior to the manual coding. The utilization of the algorithm offers significant savings in time, human resources and cost compared to a singular manual coding approach.

Conclusions The coding and harmonization of this multicohort data demonstrates the value of the ACA-NOC algorithm, while increasing the utility of the CanPath data and research related to occupational health. Future research may involve comparisons between CanPath and international cohorts.

\section{0-299 AN APPLICATION OF A PSEUDOLIKELIHOOD APPROACH IN A COUNTER-MATCHED STUDY OF BLADDER CANCER IN A COHORT OF STEEL WORKERS EXPOSED TO METALWORKING FLUID MISTS}

${ }^{1}$ Michel Grzebyk, Mohamed Arnouss, Regis Colin, Eve Bourgkard, Pascal Wild. ${ }^{1}$ French National Research and Safety Institute, France

\subsection{6/OEM-2021-EPI.81}

Objectives To compare partial likelihood and pseudolikelihood approaches in a nested case-control study under countermatching design in the presence of time varying-covariates.

Methods Within a prospective cohort of 17603 French steel workers, cases were newly diagnosed with a histologically-confirmed carcinoma of the bladder in 2006-2012. Three controls per case matched on age at diagnosis were randomly selected following a countermatched sampling scheme using a four-strata surrogate time-varying exposure to metalworking fluid (MWF) mists covariate, assessed by a job-exposure matrix. Cases $(n=84)$ and controls $(n=251)$ provided information during questionnaire interviews concerning smoking history and occupational exposures history that were compiled by experts to assess occupational exposure to straight MWFs, 